\title{
Integrin B6 - A Potential Marker for the Early Malignant Transformation in Prostate Cancer
}

\author{
A. Otsetov ${ }^{1 \#}$, Linlin Gao ${ }^{1,2}$, Evelina Wang ${ }^{2 \#}$ and Wanzhong Wang ${ }^{1 *}$ \\ ${ }^{1}$ Department of Medical Biosciences, Pathology, Umeå University, Sweden \\ ${ }^{2}$ National Institute of Biological Sciences, Beijing, China
}

Received: June 20, 2016; Accepted: : July 6, 2016; Published: July 8, 2016

*Corresponding author: Wanzhong Wang, Department of Medical Biosciences, Pathology, Building 6M, Umeå University Umeå, Sweden, SE-901 85, Phone: +46 90785 1508; Mobile: +46 72321 74 56; E-mail: wanzhong.wang@gmail.com

\begin{abstract}
Background: Integrins play important role in Prostate Cancer (PCa) initiation and progression and also may serve as biomarkers in its detection.

Objective: To evaluate and assess the $\alpha v, \beta 6$ and $\alpha v \beta 6$-integrin expression in different prostatic lesions, as well as to explore the prostate malignant transformation.

Design, setting, and participants: Immunohistochemical assessment of the integrin $\alpha v, \beta 6$ and $\alpha v \beta 6$ expression in 48 prostatectomy and 11 bone metastasis samples was performed. The expression was examined in normal, atrophic, Low-Grade Intraepithelial Neoplasia (LGPIN), High-Grade Intraepithelial Neoplasia (HGPIN) and PCa tissues.
\end{abstract}

Outcome measurements and statistical analysis: Kruskal-Wallis tests were used to evaluate the integrins $\alpha v, \beta 6$ and $\alpha v \beta 6$ expression and their associations along with clinical variables and distribution in tissues.

Results: $\alpha v$ highest expression was detected in bone metastasis samples. Integrin $\beta 6$ had weak staining in normal tissues, with a peak in LGPIN and HGPIN. The majority of PCa demonstrated integrin $\beta 6$ negative/weak staining. Integrin $\beta 6$ in the atrophy-LGPIN, -HGPIN and -PCa merging lesions was elevated. The expression of $\alpha \mathrm{v} \beta 6$ was remarkable in the prostatic atrophy and PCa bone metastasis.

Conclusions: Our results suggest that the prostate atrophy can be another candidate precursor of PCa. The elevated expression of integrin $\beta 6$ in LGPIN provides new evidence about the role of LGPIN as a risk factor for PCa. Our data shed light on possible role of the integrin $\beta 6$ as a specific biomarker for the early malignant prostatic development and warrant further investigations in the intricate process of prostatic neoplasia.

Keywords: Atrophy; Integrins; Precursor; Low-grade intraprostatic neoplasia; Prostate cancer

\section{Abbreviations}

IHC: Immunohistochemistry; GS: Gleason Score; H\&E: Hematoxylin/Eosin; HPF: High Field Power; $\mathrm{H}_{2} \mathrm{O}_{2}$ : Hydrogen Peroxide; DAB: 3, 3'-Diaminobenzidine Tetra Hydrochloride; PBS: Phosphate Buffered Saline; LGPIN: Low-Grade Intraepithelial
Neoplasia; HGPIN: High-Grade Intraepithelial Neoplasia; PSA: Prostate Specific Antigen; Pca: Prostate Cancer; PIA: Proliferative Inflammatory Atrophy; TRUS: Transrectal Ultrasound; SD: Standard Deviation; WHO: World Health Organization

\section{Introduction}

Prostate Cancer (PCa) is the most common neoplasm among men worldwide and second leading cause of cancer death after the lung cancer [1]. It is also a significant economic burden, associated with impaired quality of life [2]. Despite of significant progress in diagnosis and treatment, the PCa etiology is not completely elucidated. Detected at early stage, PCa is curable and the prompt diagnosis is essential, improving the efficiency of treatment. Therefore, the need of identification of the earliest step in malignant transformation of PCa from its non-malignant precursors and developing of new biomarkers is imperative. Prostate cancer is a step-wise process that develops from its forerunners or precursors. These defined states arise through multiple transformations in normal cell functions $[3,4]$. To date, between several proposed forerunners, only the HGPIN appears to be a true precursor of prostate cancer that can develop into invasive cancer. In contrast, LGPIN is not believed to be associated with PCa risk and its presence on the prostate biopsy is not yet reported [4]. Since the relation inflammation-cancer was demonstrated as mechanism, contributing to carcinogenesis, another possible PCa precursor, linked to chronic inflammationthe Proliferative Inflammatory Atrophy (PIA), has been [5]. PIA includes changes predominantly found in the peripheral zone of the prostate [6] caused by many factors, leading to prostatic injury with subsequent PCa development $[7,8]$. The atrophic epithelial cells in the merging HGPIN / PCa lesions demonstrate intermediate phenotype with a higher proliferative and low apoptosis rate, expressing specific proteins [9-11]. A critical point during prostate cancer progression is the loss of Basal Cell Membrane (BM), which is a hallmark of malignant progression.

This mechanism is very intricate and includes multiple alterations in the cellular homeostasis. Among the alterations described critical point to malignant invasion take place aberrant 
interactions glandular epithelial cells and the extracellular matrix, mediated by Cell Adhesion Molecules (CAMs) [12-17]. In the prostate cancer, tumor cells express an abnormal integrin repertoire and are surrounded by abnormal ECM. CAMs are divided in 4 categories, including cadherins, integrins, selectins and members of the immunoglobulin super family [12]. Integrins are transmembrane glycoprotein receptors for ECM proteins, composed by $\alpha$ and $\beta$ subunits and a combination of which determines a different specifies and function [12]. Currently 24 members of the integrin family, $18 \alpha$ and $8 \beta$ subunits are described [18]. Studies demonstrated their role in-between epithelial cells and ECM interactions in the tumor genesis of PCa $[17,18]$. The deregulation of the integrin expression during PCa progression to its androgen-independent form has been described. Most $\alpha$ and $\beta$ subunits were demonstrated to be down regulated in the prostate cancer. Among the integrin family, $\alpha v$, $\beta 6$, and $\alpha \nu \beta 6$ types are stated as specific players in PCa but little is known about their effect in the precursor lesions and their expression in these lesions is less well-investigated.

This study provides new evidence of integrins $\alpha v, \beta 6$, and $\alpha v \beta 6$ expression in human prostate tissues and their association with PCa initiation and development. We also reported a new finding of significant expression of the integrin $\beta 6$ in LGPIN lesions and the plausible role of Integrin $\beta 6$ as a possible biomarker for early PCa detection.

\section{Material and Methods}

\section{Tissue samples and data collection}

Specimens from 48 prostatectomies performed for prostatic adenocarcinoma were obtained from Norrland's University hospital, Umeå, Sweden and 11 PCa bone metastasis samples from Sahlgrenska University hospital, Gothenburg, Sweden were used. Data like age, PSA, Gleason score and pathologic stage were extracted from clinical database. Histological diagnosis of prostate cancer was based on H\&E-stained sections. The Gleason system was used for histological grading. A primary and secondary Gleason grade (2-5) was determined for each tumour and the Gleason sum was scored.

\section{Tissue processing, IHC staining and expression detec- tion}

After pathological examination all tissues were fixed in formalin and processed routinely through graded alcohols to paraffin blocks.

\section{Antibodies}

The following antibodies were used in this study: Integrin $\alpha v$ (working dilution 1:50, catalog no.sc-9969, Santa Cruz Biotechnology, Inc.), Integrin $\beta 6$ (working dilution 1:50, catalog no. sc-6632, Santa Cruz Biotechnology, Inc.), anti-integrin $\alpha v \beta 6$ monoclonal antibody (working dilution 1:50, Millipore), and the secondary antibody Real ${ }^{\mathrm{TM}}$ EnVisioin HRP rabbit/ mouse (Dako).

\section{Immunohistochemical (IHC) staining}

The serial sections were mounted on glass slides (Fisher Scientific, Rochester, NY). All IHC staining were completed using
Dako Real ${ }^{\mathrm{TM}}$ EnVisioin system kit (Dakocytomation, Carpinteria). Briefly, after deparaffinization and rehydration of tissues, the antigen retrieval was performed in a microwave oven in 0.01 Mcitrate buffer (pH 6.0) for 10 min. Next, Ethanol 2\% and $\mathrm{H}_{2} \mathrm{O}_{2}$ were used to inactivate endogenous peroxidise activity. Diluted primary antibodies against integrin $\alpha v, \beta 6$ and $\alpha v \beta 6$ were incubated overnight on slides at $4^{\circ} \mathrm{C}$ and secondary antibody was incubated subsequently at room temperature for 30 minutes. The IHC was labelled by applying 3, 3'-diaminobenzidine tetra hydrochloride (DAB). All slides were counterstained with Mayer haematoxylin and observed under microscope. Negative control was performed with the substitution of the primary antibodies by PBS.

\section{Integrin $\alpha v, \beta 6$ and $\alpha v \beta 6$ IHC expression scores}

Prostatectomy lesions samples were classified as follows: normal-appearing acini, atrophy, LGPIN, HGPIN and PCa. The definition of prostatic atrophy, LGPIN, and HGPIN is based as previously defined $[4,5]$. The pathological diagnostic standards and Gleason's grades were in accordance with WHO 2003 guidelines. The positive immunostaining was identified by the presence of marked brown/ yellow color in cytoplasm of prostate epithelia cells. The evaluation of integrin expression scores was accomplished under microscope with a set at $\mathrm{x} 100$ magnification. Each slide was observed and scored independently and the scores were summed. We stated the final score as $\mathrm{M}$, calculated as extension score (E) multiplied by intensity score (I). The intensity was scored ( 0 (negative), 1(weak), 2 (moderate) to 3(strong). The extension of stained cells (E) was recorded in a 4-tiered scale: 0: (none); $1(<1 / 3) ; 2$ (between $1 / 3$ and 2/ 3); and $3(>2 / 3)$. The final score $(M)$ was used for statistics (range from 0 to 9 ).

\section{Expression of Integrin $\beta 6$ in various prostatic lesions}

The integrin $\beta 6$ expression score in various prostatic lesions was scored under high power fields $(\times 20)$ separately. Thirty ocular measuring fields have been chosen randomly for every section.

\section{Statistical Analysis}

Statistical analyses were performed with SPSS version 21 for Windows. The final scores (M) of $\alpha v, \beta 6$ and $\alpha v \beta 6$ expression and their associations with the age, PSA, Gleason score and pathologic stage were analyzed with Mann-Whitney test. The comparison of $\alpha v, \beta 6$ and $\alpha v \beta 6$ integrins expression was assessed using Kruskal-Wallis test. Chi-square test was performed to analyse the expression of Integrin $\beta 6$ in various prostatic lesions and $p<0.05$ was considered statistically significant.

\section{Results \\ Demographic and clinical features}

Patient characteristics are reported in Table 1. Mean age was 65 years (range: $52-81$ ) and PSA level was $8.8 \mathrm{ng} / \mathrm{ml}$ (range: 2.3-20). Significances were calculated compared among age, PSA, Gleason score and pathologic stage in expression final scores (M) of different integrins $(\alpha v, \beta 6, \alpha v \beta 6)$. For the statistical analysis in 
our sample size tumours were categorized as low (Gleason score $\leq 7 ; n=28$ ), and high grade (Gleason score $>7 ; n=20$ ).

\section{Various Expression of Integrin $\alpha v, \beta 6$ and $\alpha v \beta 6$ in dif- ferent prostatic lesions}

We assessed the expression of Integrin $\alpha v, \beta 6$ and $\alpha v \beta 6$ in the normal-appearing acini $(\mathrm{n}=22)$, atrophy $(\mathrm{n}=48)$, LGPIN ( $=29)$, HGPIN $(n=42)$ and PCa $(n=45)$. All samples contained atrophy component. The expression of these integrins is shown in Table 2. Scattered staining was found in macrophages. None of these Integrins were expressed in myofibrous stromal cells.

\section{Increased integrin $\alpha v$ expression in high PCa grade and PCa bone metastasis}

Integrin $\alpha v$ was negative or weakly stained in the normalappearing acini (Mean \pm SD, $0.2 \pm 0.7$ ); increased immunostaining was seen in the atrophy $(1.0 \pm 1.3)$, LGPIN $(1.1 \pm 1.3)$, and HGPIN (1.9 \pm 1.4$)$, and in PCa $(1.7 \pm 1.7)$ as well. In the PCa samples, the expression of Integrin $\alpha \mathrm{v}$ in relation to different Gleason grades was analyzed (Table 3). Integrin $\alpha v$ levels were increased in higher Gleason grade areas. In the PCa bone metastasis samples, Integrin $\alpha v$ showed highly intensive cytoplasm immunostaining (Mean \pm SD 8, $0 \pm 1.8$ ), which was significantly higher than in the primary $\mathrm{PCa}$, irrespective of their Gleason grade $(P<0.001)$ (Figure 1; Table 3, 4).

\section{Integrin $\beta 6$ expression in the prostatic atrophy and merging lesions}

Compared to Integrin $\alpha v$, integrin $\beta 6$ was expressed in a lesser extent in the PCa tissues, especially in those with high Gleason grade (0.4 \pm 0.8 in Gleason 4 area and 0 in Gleason 5) and metastasis cancer $(0.6 \pm 1.4)$, respectively. In contrast, its

\begin{tabular}{|c|c|c|c|c|c|c|c|}
\hline \multirow[b]{2}{*}{ Variables } & \multicolumn{3}{|r|}{$\alpha \mathbf{v}$} & \multicolumn{2}{|r|}{$\beta 6$} & \multicolumn{2}{|r|}{$\alpha v \beta 6$} \\
\hline & & $\begin{array}{l}\text { Mean } \pm \\
\text { SD }\end{array}$ & $\begin{array}{l}p^{a}- \\
\text { value }\end{array}$ & $\begin{array}{l}\text { Mean } \pm \\
\text { SD }\end{array}$ & $\begin{array}{l}p^{a}- \\
\text { value }\end{array}$ & $\begin{array}{l}\text { Mean } \pm \\
\text { SD }\end{array}$ & $\begin{array}{l}p^{a-} \\
\text { value }\end{array}$ \\
\hline \multirow[b]{2}{*}{ Age (years) } & $<65$ & $2.3 \pm 1.9$ & 0.033 & $\begin{array}{l}3.5 \pm \\
2.2\end{array}$ & 0.192 & $\begin{array}{l}3.6 \pm \\
3.1\end{array}$ & 0.644 \\
\hline & $\geq 65$ & $1.1 \pm 1.4$ & & $\begin{array}{l}2.6 \pm \\
1.3\end{array}$ & & $\begin{array}{l}2.9 \pm \\
2.2\end{array}$ & \\
\hline \multirow{2}{*}{ PSA } & $\begin{array}{l}< \\
8,8\end{array}$ & $1.8 \pm 1.4$ & 0.881 & $\begin{array}{l}3.5 \pm \\
2.1\end{array}$ & 0.033 & $\begin{array}{l}3.6 \pm \\
2.6\end{array}$ & 0.155 \\
\hline & $\begin{array}{l}\geq \\
8,8\end{array}$ & $1.9 \pm 2.0$ & & $\begin{array}{l}2.4 \pm \\
1.2\end{array}$ & & $\begin{array}{l}2.6 \pm \\
2.9\end{array}$ & \\
\hline \multirow{3}{*}{$\begin{array}{l}\text { Gleason } \\
\text { score }\end{array}$} & $\leq 6$ & $1.8 \pm 2.0$ & 0.554 & $\begin{array}{l}3.4 \pm \\
2.5\end{array}$ & 0.784 & $\begin{array}{l}3.4 \pm \\
1.8\end{array}$ & 0.833 \\
\hline & 7 & $1.5 \pm 1.4$ & & $\begin{array}{l}2.9 \pm \\
1.7\end{array}$ & & $\begin{array}{l}3.2 \pm \\
3.0\end{array}$ & \\
\hline & $\geq 8$ & $2.6 \pm 2.6$ & & $\begin{array}{l}3.2 \pm \\
1.5\end{array}$ & & $\begin{array}{l}3.1 \pm \\
2.3\end{array}$ & \\
\hline \multirow{2}{*}{$\begin{array}{l}\text { Pathologic } \\
\text { stage }\end{array}$} & $\mathrm{T} 2$ & $2.0 \pm 1.9$ & 0.339 & $\begin{array}{l}3.2 \pm \\
2.1\end{array}$ & 0.770 & $\begin{array}{l}3.2 \pm \\
2.7\end{array}$ & 0.861 \\
\hline & T3 & $1.4 \pm 1.4$ & & $\begin{array}{l}2.8 \pm \\
1.4\end{array}$ & & $\begin{array}{l}3.2 \pm \\
2.7\end{array}$ & \\
\hline
\end{tabular}

SD: Standard Deviation; PSA: Prostate-Specific Antigen; ${ }^{a}$ Mann-Whitney test
Table 2: Comparison of $\alpha v, \beta 6$ and $\alpha v \beta 6$ antibodies expression in various prostatic lesions

\begin{tabular}{|c|c|c|c|c|c|c|}
\hline \multirow[b]{2}{*}{$\begin{array}{l}\text { Prostate } \\
\text { lesions }\end{array}$} & \multicolumn{2}{|r|}{$\alpha \mathbf{v}$} & \multicolumn{4}{|c|}{$\beta 6_{\alpha v \beta 6}$} \\
\hline & number & $\begin{array}{l}\text { Mean } \pm \\
\text { SD }\end{array}$ & number & $\begin{array}{l}\text { Mean } \pm \\
\text { SD }\end{array}$ & number & $\begin{array}{l}\text { Mean } \pm \\
\text { SD }\end{array}$ \\
\hline Normal & 22 & $0.2 \pm 0.7$ & 22 & $1.2 \pm 1.2$ & 22 & $0.2 \pm 0.7$ \\
\hline Atrophy & 48 & $1.0 \pm 1.3$ & 48 & $3.0 \pm 1.8$ & 48 & $3.2 \pm 2.7$ \\
\hline Low-PIN & 29 & $1.1 \pm 1.3$ & 29 & $4.5 \pm 1.9$ & 29 & $1.2 \pm 1.9$ \\
\hline High-PIN & 42 & $1.9 \pm 1.4$ & 42 & $5.2 \pm 2.2$ & 42 & $1.3 \pm 1.5$ \\
\hline PCa & 45 & $1.7 \pm 1.7$ & 45 & $1.5 \pm 1.9$ & 45 & $0.6 \pm 1.1$ \\
\hline pb-value & & $<0.001$ & & $<0.001$ & & $<0.001$ \\
\hline
\end{tabular}

All the 48 samples contained atrophy component. The highest expression of $\alpha v$ integrin is in the HGPIN lesions; $\beta 6$ is significantly expressed in the HGPIN but also in LGPIN lesions; $\alpha \nu \beta 6$ is expressed mostly in the atrophy lesions. None of these integrins is expressed in myo fibrous stromal cells. SD: Standard Deviation; PCa: Prostate Cancer, ${ }^{\mathrm{B} K r u s k a l-W a l l i s ~ t e s t ~}$

Table 3: Comparison of the integrins expression in Gleason 3, 4, 5 grades and bone metastasis lesions

\begin{tabular}{|c|c|c|c|c|c|c|}
\hline & \multicolumn{2}{|r|}{$\alpha \mathbf{v}$} & \multicolumn{2}{|r|}{$\beta 6$} & \multicolumn{2}{|r|}{$\alpha v \beta 6$} \\
\hline PCa & number & $\begin{array}{l}\text { Mean } \pm \\
\text { SD }\end{array}$ & number & $\begin{array}{l}\text { Mean } \pm \\
\text { SD }\end{array}$ & number & $\begin{array}{l}\text { Mean } \pm \\
\text { SD }\end{array}$ \\
\hline GG-3 & 36 & $1.3 \pm 1.5$ & 36 & $2,3 \pm 2,2$ & 36 & $0,6 \pm 1,2$ \\
\hline GG-4 & 28 & $2.4 \pm 2.0$ & 28 & $0,4 \pm 0,8$ & 28 & $0,9 \pm 1,4$ \\
\hline GG-5 & 2 & $4.5 \pm 2.1$ & 2 & 0 & 2 & $1,5 \pm 2,1$ \\
\hline $\begin{array}{l}\text { Bone } \\
\text { Metastasis }\end{array}$ & 11 & $8,0 \pm 1,8$ & 11 & $0,6 \pm 1,4$ & 11 & $5,1 \pm 3,1$ \\
\hline pb-value & & 0.001 & & $<0.00$ & & $<0.001$ \\
\hline
\end{tabular}

Integrin $\alpha v$ demonstrates increased reaction in PCa, especially in the higher Gleason areas. Integrin $\alpha \nu$ is overexpressed in all 11 bone metastasis samples, which is essentially higher than in any other GS cancers.

SD: Standard Deviation; PCa: Prostate Cancer; GG: Gleason Grade; ${ }^{B}$ Kruskal-Wallis test

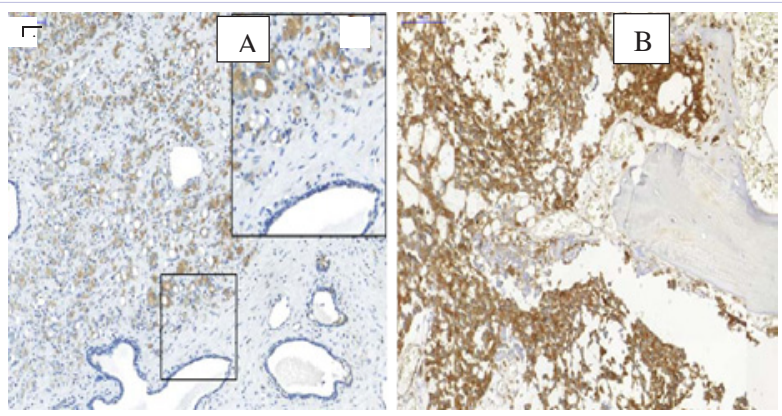

Figure 1: Integrin $\alpha v$ expression in human prostate cancer. (A) PCa, Gleason grade 4 (B) PCa bone metastasis

expression appeared stronger in atrophy ( $3.0 \pm 1.8)$, LGPIN ( $4.5 \pm$ 1.9), HGPIN (5.2 \pm 2.2$)$ and in the merging-lesions (Figure 2, Table $3)$. We next analysed the Integrin $\beta 6$ expression in the atrophymerging lesions (Table 4). Five to ten high power field (x20) per case were reviewed to assess the expression in the pure atrophic 
and merging-LGPIN, -HGPIN and -PCa lesions. The Integrin $\beta 6$ expression in the atrophic epithelial and merging- atypical cells was also scored. The atrophic component in atrophy-LGPIN and -HGPIN merging lesions showed positive integrin $\beta 6$ staining $12.2 \%(11 / 90)$ in LGPIN and 4.8\% (2/41) in HGPIN, respectively. Conversely, the atypical cells in these entities demonstrated positive immunostaining up to $88.9 \%(80 / 90)$ in LGPIN and 97.6\% (40/41) in HGPIN $(p<0.001)$. However, in all 6 observed atrophy-PCa merging areas, the malignant elements showed complete negative signalling for integrin $\beta 6$.

\section{Integrin $\alpha v \beta 6$ showed high expression in prostatic at- rophy}

We assessed the integrin $\alpha \nu \beta 6$ expression in different prostatic lesions (Figure3, Table 2). The highest expression levels of $\alpha v \beta 6$ were found in the atrophic changes (Mean \pm SD; $3.2 \pm$ $2.7)$, compared to normal $(0.2 \pm 0.7)$, LGPIN (1.2 \pm 1.9$)$, HGPIN $(1.3 \pm 1.5)$, and PCa $(0.6 \pm 1.1)(p<0.001)$. Further $\alpha v \beta 6$ analysis in the primary $\mathrm{PCa}$ and bone metastasis samples demonstrated its highest score in the bone specimens $(5.1 \pm 3.1)$, opposite to lower scores in the primary PCa $(p<0.001)$

\section{Discussion}

The integrins, including $\alpha v, \beta 6$, and $\alpha v \beta 6$, play a vital role in the cross-talk between the cell and extracellular matrix, enhancing the growth, migration, invasion and metastasis of cancer cells $[18,19]$, their role in the normal prostate, as well as the PCa development has been demonstrated in many studies. Studies have shown that the normal prostate expresses integrins, such as $\alpha v \beta 1$ and $\beta 4$. Among the $\beta$ subunits, $\beta 1 c$ and $\beta 1$ a are expressed in normal prostatic epithelium.

A critical point in prostate cancer progression is the loss of basal cell membrane, which is strongly associated with changes in the integrin levels and their abnormal expression is a one of the characteristic features of the prostatic carcinoma. In contrast to normal prostate, the expression of integrins in PCa demonstrates some specific features. For example, higher Gleason score was correlated with low or negative expression of subunit $\alpha 3$. Similarly, the expression of subunit $\alpha 6$ decreases with the increase of the histologic grade of PCa [12]. $\alpha 2, \alpha 4, \alpha 5$

Table 4: Expression of Integrin $\beta 6$ in atrophy, PIN and merging-lesions

\begin{tabular}{|l|l|l|l|l|l|l|l|}
\hline & \multicolumn{3}{|c|}{ Atrophic epithelium } & \multicolumn{3}{|c|}{ Atypical component } \\
\hline Prostatic lesions & - & + & ++ & - & + & ++ \\
\hline Atrophy & 218 & 121 & 2 & - & - & - \\
\hline $\begin{array}{l}\text { Atrophy merging } \\
\text { with LGPIN }\end{array}$ & 79 & 11 & 0 & 10 & 64 & 16 \\
\hline $\begin{array}{l}\text { Atrophy merging } \\
\text { with HGPIN }\end{array}$ & 39 & 2 & 0 & 1 & 15 & 25 \\
\hline $\begin{array}{l}\text { Atrophy merging } \\
\text { with } \\
\text { PCa }\end{array}$ & 3 & 2 & 1 & 6 & 0 & 0 \\
\hline
\end{tabular}

Integrin $\beta 6$ is weakly expressed in the PCa tissues, especially in those with high Gleason grade, and metastasis cancer. However, its expression is much higher in the atrophy, LGPIN, HGPIN, and merging lesions. LGPIN: Low-Grade Prostatic Intraepithelial Neoplasia; HGPIN: HighGrade Prostatic Intraepithelial Neoplasia; PCa: prostate cancer

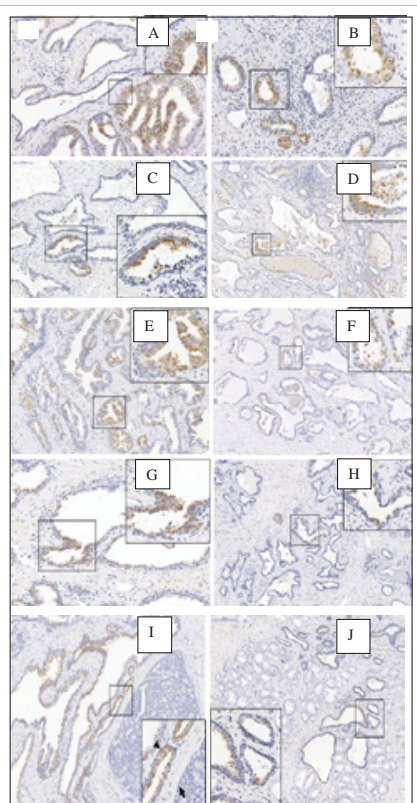

Figure 2: Integrin $\beta 6$ expression various prostatic lesions. Strong cytoplasm immunostaining in high grade PIN (A and B) and the HGPIN components in HGPIN-merging lesion (C and D). Cytoplasm immunostaining in low grade PIN ( $\mathrm{E}$ and $\mathrm{F}$ ) and the LGPIN-merging lesions (G and $\mathrm{H}$ ). To note, PCa cells show negative/weak positive immunostaining (I and J).

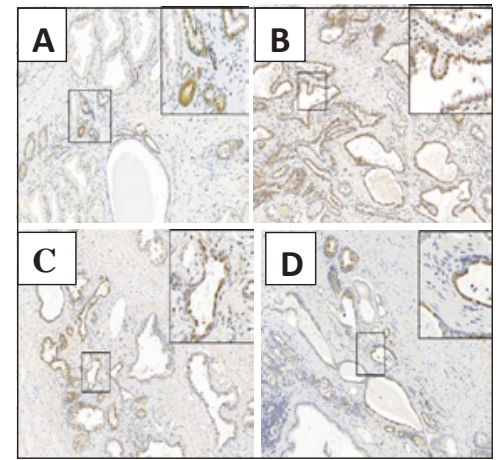

Figure 3: Integrin $\alpha v \beta 6$ expression in prostate atrophic lesions. Integrin $\alpha v \beta 6$ show cytoplasm staining. To note, prostate cancer shows negative Immunostaining.

and $\alpha \nu$-subunits are down-regulated in prostatic carcinoma and some subunits such $\alpha \mathrm{Ii} \beta$ are specifically expressed in PCa but not in normal tissue $[12,17,18]$. $\beta 1 \mathrm{c}$ that is normally expressed in normal prostate, has been found also in the PCa. In addition, studies have shown that expression of $\beta 1 \mathrm{~A}$ can promote cell proliferation [12-19]. Up regulation of $\beta 3$ and $\beta 6$ subunits was described in PCa, as well as the specific expression of $\alpha v \beta 3$ and $\alpha v \beta 6$ in PCa, compared to normal prostate, where they are not detected [12]. As unique expression feature was shown also the $\alpha 2$ subunit, which is up regulated in lymph nodes metastasis, compared with primary lesions, where it is not

Here, we assessed the expression of $\alpha v, \beta 6$ and $\alpha v \beta 6$ in different prostatic tissues as well as in the bone metastasis samples. 


\section{Integrin $\alpha v$}

In our study we stated an increased trend of the integrin $\alpha v$ expression from PIA to HGPIN, with significant peak in the bone metastases samples; its relation to Gleason grade also was shown. Our results are consistent with previous studies, focused on the $\alpha \nu$ role in promotion and development of PCa bone metastasis [16-19]. $\alpha v$ integrins also play an important role in developmental angiogenesis. Before dissemination, cancer cells become motile and detach from the primary tumor, which is accompanied by $\alpha \nu$ elevation in the primary tumor and overexpression in bone metastases [19-23]. In a study performed with primary PCa and lymph node metastasis samples, the integrin $\alpha v$ was abnormal in the primary neoplasms, whereas in the lymph node metastases its levels were significantly reduced. The global down regulation of $\alpha \mathrm{v}$ was demonstrated as a sign of advanced disease and poor prognostic factor [20-24]. Similarly, the unregulated integrin $\alpha v$ expression was found in higher grade tumors with lymph node metastases [24-26], demonstrating its various expression in different types of neoplasm's.

\section{PIA and LGPIN as potential PCa precursors}

PCa develops from lesions, preceding its development by many years. There is no consensus on possible precursors and presently only HGPIN is considered as a PCa driver. There are several criteria to consider a prostatic lesion as premalignant [4-28]. PIA is suggested as another precursor, contributing to PCa [6-30], which is frequently found in the peripheral zone, adjacent to HGPIN and cancer areas [25,26]. Genetic pathway in-between PIA, HGPIN, and PCa also has been demonstrated [631]. Unlike HGPIN, LGPIN is believed do not contribute to PCa and is no longer reported as finding [29-34]. However, the likely outcome of isolated LGPIN lesions in prostate biopsies remains unclear. In a study performed with younger men, PIN changes were seen even in their 20's and 30's. Most foci were LGPIN, with increasing frequency of HGPIN with advancing age [3234]. Another analysis demonstrated $30 \%$ risk of PCa on repeat sextant biopsy in the LGPIN cohort [31]. Albeit HGPIN is the only known currently accepted PCa precursor, in this study there was at least a comparable percentage of patients who had cancer on repeat biopsies [31]. This finding is highlighted as worrying since LGPIN is not considered as a risk factor. The authors showed the predictive value for PCa to be equal with both, isolated LGPIN and HGPIN at biopsies and LGPIN was demonstrated as a risk factor for PCa progression. The conclusion made is that chemoprevention can be beneficial not only in HGPIN, but in LGPIN.

\section{Integrin $\beta 6$ subunit}

In the normal prostate $\beta 6$ levels are absent or expressed in a lesser extent, but increased in cases of BPH [24] and significantly elevated in PCa [32-37]. In our study the $\beta 6$ levels in the normal prostate were insignificant, and then they gradually increased in PIA and reached a peak in both, LGPIN and HGPIN, and declined in PCa. The $\beta 6$ expression in atrophic and merging lesions indicates the plausible mechanism of PCa transformation from its precursors. Key finding is the significant expression of $\beta 6$ in LGPIN, suggesting its malignant potential in PCa development.
Limited data shows the possible outcome of LGPIN on prostate biopsy and our results cautiously suggest the likely malignant potential of LGPIN, suggesting the need for closer follow-up in patients with LGPIN at the initial biopsy. PIN lesions don't affect PSA levels and are detectable only by prostate biopsies, frequently associated with a risk of infections, requiring hospitalization and antibiotic treatment. However, studies have demonstrated a link between PIN grades and the level of disruption of the basal cell layer, accompanied by expression of functional molecules, specific to every stage of tumor progression [5]. Therefore, our data can contribute to development of new markers, useful for the selection of patients at risk that may need closer follow-up and re-biopsy with those, in whom this intervention can be safely skipped.

\section{Integrin $\alpha v \beta 6$}

Was strongly expressed in our atrophic and bone metastasis samples $\alpha \nu \beta 6$ was shown to be implicated in progression of number of cancers, promoting the invasion and metastasis [43]. However, in the PCa and its precursors $\alpha \nu \beta 6$ has been investigated in a lesser extent. Studies showed the $\alpha v \beta 6$ high expression in basal cells in the HGPIN, normal tissue adjacent to tumors [38], as well as in the areas of inflammation and proliferative inflammatory atrophy [37]. Once the expression of this integrin was associated with inflammation and its $\beta 6$ subunit is increased in PCa, the $\alpha v \beta 6$ has also been implicated in controlling PCa growth in conjunction with the androgen receptor. The elevation of $\alpha v \beta 6$ indicates advanced disease, associated with poor prognosis. Similarly to $\beta 6, \alpha v \beta 6$ levels are inducible in PCa and bone metastasis and absent or insignificant in the normal prostatic tissues [39-42]. The association between $\alpha \nu \beta 6$ and the inflammation, as well as a link between chronic inflammation and PCa initiation has been shown $[35,36]$. TGF- $\beta$ up regulates expression of the integrins and there is an extensive cross-talk between TGF- $\beta$ and the large repertoire of these integrins, including $\alpha \nu \beta 6$ [29-36]. TGF also regulates EMT, where cells acquire the invasive phenotype that is required for metastasis [37-40]. EMT is associated with increased $\alpha v$ expression. In cancer, alongside with the other integrins, $\alpha v \beta 6$ has been shown to be important in allowing EMT to take place in the metastatic process and $\alpha v \beta 6$ is also induced in this process [41-43]. The role of integrin $\alpha_{v} \beta_{6}$ in the relation inflammationcancer was demonstrated in the POET study [44], where $\alpha v \beta 6$ is expressed in both, inflammation and cancer and not in normal tissue, thus suggesting not only the link between inflammation and PCa [43]. Furthermore, besides the $\alpha \nu \beta 6$ expression, induced by the inflammation in this study, this integrin has been proposed to support metastasis by activating TGF- $\beta 1$ that is associated with metastasis [42]. In our study $\alpha \nu \beta 6$ is mainly expressed in atrophic lesions, supporting the hypothesis of the role of atrophy as precursor lesion but also in the bone metastasis samples.

Studies about the role of integrins in PCa developing, led to conduction of several ongoing clinical trials, evaluating the efficacy of integrin antagonists as prostate cancer therapeutics [12]. Thus, the role of Cilengitide, a cyclic Arg-Gly-Asp peptide that inhibits $\alpha \nu \beta 3$ and $\alpha \nu \beta 5$, has been demonstrated. CNTO 95, as well as MEDI-522, monoclonal antibodies against $\alpha \nu$ integrin 
also have been suggested as promising therapeutic agents [12] Therefore, our findings can also have far-reaching implications for the management and chemo-prevention of $\mathrm{PCa}$, inviting future studies to explain the malignant transformation. The early identification of PCa risk patients will help to tailor the best therapeutic approach [45] and designing novel therapeutic approaches based on inhibiting integrin expression may be a promising strategy.

We consider as a major limitation of this study the relatively small sample size and the clinical significance of data requires further investigations to validate recent results.

\section{Conclusions}

In summary, the present data identify the integrins $\alpha v, \beta 6$ and $\alpha v \beta 6$ action in prostate cancer precursors. Prostate cancer is a chronic, multi-step disease that arises from its premalignant precursors and to date; HGPIN is the only accepted PCa precursor. The expression of integrins $\alpha v, \beta 6$ and $\alpha v \beta 6$ reveals new evidence of malignant $\mathrm{PCa}$ initiation and transformation. In addition, the elevation of $\beta 6$-subunit in the LGPIN may suggest more malignant potential than previously believed. Our findings can give new directions in the personalized therapy of PCa and may contribute to the therapeutic optimization of existing trials for $\mathrm{PCa}$. This is a novel study for the integrins role in PCa precursors and molecular pathways by which integrins contribute to $\mathrm{PCa}$ initiation and progression need to be further elucidated.

\section{Disclosure}

This study was supported by ALF - Västerbotten, Lion Cancer Fond, and Umeå University. The study received institutional review board approval by the Ethical Board at the Norrland's University Hospital, Umeå, Sweden under the protocol Dnr 2010/366-31M

\section{Acknowledgments}

This investigation was supported by grants from the Cancer Research Foundation in Northern Sweden) Cancerforskningsfonden I Norrland/Lions CancerforskningsfondUmeå, Sweden, LP 15-2096, 2015.

We also would like to thank the Departments of Pathology at the Norrland's University Hospital, Umeå, Sweden and Sahlgrenska University Hospital, Gothenburg, Sweden

\section{References}

1. Siegel RL, Miller KD, Jemal A. Cancer statistics. CA Cancer J Clin. 2016;66(1):7-30. doi:10.3322/caac.21332.

2. Fradet $Y$, Klotz L, Zlotta A, Trachtenberg J. The burden of prostate cancer in Canada. Can Urol Assoc J. 2009;3(3 Suppl 2): S92-S100.

3. Goel HL, Li J, Kogan S, Languino LR. Integrins in prostate cancer progression. Endocr Relat Cancer. 2008;15(3):657-64. doi: 10.1677/ ERC-08-0019.

4. Botswick DG, Cheng L. Precursors of prostate cancer. Histopathology. 2012;60(1):4-27. doi: 10.1111/j.1365-2559.2011.04007.x.

5. De Marzo AM, Marchi VL, Epstein JI, Nelson WG. Proliferative inflammatory atrophy of the prostate: implications for prostatic carcinogenesis. Am J Pathol. 1999;155(6):1985-92.
6. De Marzo AM, Platz EA, Sutcliffe S, Xu J, Grönberg H, Drake CG, et al. Inflammation in prostate carcinogenesis. Nat Rev Cancer. 2007;7(4):256-69.

7. Elkawaji JE. The role of inflammatory mediators in the development benign prostatic hyperplasia and prostatic carcinoma. Research and Report Urol. 2013;5:1-10.

8. Bechis SK, Otsetov AG, Olumi AF, Wanq Z, Vanqel MG, Tabatabaei S, et al. Age and obesity promote methylation and suppression of 5-alpha reductase 2- implications for personalized therapy in BPH. J Urol. 2015;194(4):1031-7. doi: 10.1016/j.juro.2015.04.079.

9. van Laeender GJ, Gage WR, Hiks JL, Van Balken B, Schalken JA, Aalders TW, et al. Intermediate cells in human prostate epithelium are enriched in proliferative inflammatory atrophy. Am J Pathol. 2003;162(5):1529-37.

10. Wang W, Bergh A, Damber JE. Increased expression of CCAAT/ enhancer-binding protein in proliferative inflammatory atrophy of the prostate: relation with the expression of COX-2, the androgen receptor, and presence of focal chronic inflammation. Prostate. 2007. 67(11):1238-1246.

11. Wang W, Bergh A, Damber JE. Morphological transition of proliferative inflammatory atrophy to high-grade intraepithelial neoplasia and cancer in human prostate. Prostate. 2009;69(13):1378-86. doi: 10.1002 /pros.20992.

12. Drivalos A, Papatsoris AG, Chrisofos M, Efstathiou E, Dimopoulos MA. The role of the cell adhesion molecules (integrins / cadherins) in prostate cancer. International Braz J Urol. 2011;37(3):302-6.

13. Lu P, Weaver VM, Werb Z. The extracellular matrix: a dynamic niche in cancer progression. J Cell Biol. 2012;196(4):395-406. doi: $10.1083 /$ jcb.201102147.

14. Goel HL, Alam N, Johnson NS, Naved A, Lucia R L. Integrin signaling aberrations in prostatic carcinoma. Am J Transl Res. 2009;1(3):211220.

15. Suyin PC, Dickinson JL, Holloway AF. Integrins in Prostate Cancer Invasion and Metastasis. Advances in Prostate Cancer. 2013;chapter 25:621-640.

16. Pontes-J J, Reis ST, Dall'Oglio M, Neves de Oliveira LC, Cury J, Carvalho PA, et al. Evaluation of the expression of integrins and cell adhesion molecules through tissue microarray in lymph node metastases of prostate cancer. J Carcinog. 2009;8:3.

17. Hyens RO. Integrins: bidirectional, allosteric signaling machines. Cell. 2002;110(6):673-87.

18. Sutherland M, Gordon A, Shnyder S, Patterson LH, Sheldrake HM. RGD-Binding Integrins in Prostate cancer; expression patterns and therapeutic prospects against bone metastasis. Cancers 2012;(4):1106-1145. doi:10.3390/cancers4041106.

19. Goel HL, Breen M, Zhang J, Das I, AznavoorianCheshire S, Greenberg $\mathrm{NM}$, et al. beta1A integrin expression is required for type 1 insulinlike growth factor receptor mitogenic and transforming activities and localization to focal contacts. beta1A integrin. Cancer Res. 2005;65(15):6692-700.

20. Zheng DQ, Woodard AS, Fornaro M, Tallini G, Languino LR. Prostatic carcinoma cell migration via $\alpha v \beta 3$ integrin is modulated by a focal adhesion kinase pathway. Cancer Res. 1999;59(7):1655-64.

21. Gorlov IP, Byun J, Gorlova OY, Aparicio AM, Efstathiou E, Logothesis CJ, et al. Candidate pathways and genes for prostate cancer: a metaanalysis of gene expression data. BMC Med Genomics. 2009;2:48. doi: 10.1186/1755-8794-2-48. 
22. Azare J, Leslie $\mathrm{K}$, Al-Ahmadie H, Gerald W, Weinreb PH, Violette $\mathrm{SM}$, et al. Constitutively activated Stat 3 induces tumorogenesis and enhances cell motility of prostate epithelial cells through Integrin $\beta_{6}$ Mol Cell Biol. 2007;27(12): 4444-4453

23. Van der Horst G, van den Hoogen C, Buijs JT, Cheung H, Pelger RC, Lorenzon G, et al. Targeting of $\alpha(\mathrm{v})$-integrins in stem/progenitor cells and supportive microenvironment impairs bone metastasis in human prostate cancer. Neoplasia. 2011;13(6):516-25.

24. Bonkhoff $H$, Stein U, Remberger K. Differential expression of alpha6 and alpha 2 very late antigen integrins in the normal, hyperplastic, and neoplastic prostate: simultaneous demonstration of cell surface receptors and their extracellular ligands. Hum Pathol. 1993;24(3):243-8.

25. Bostwick DG, Qian J, Lian L, Michael KB. High-grade prostatic intraepithelial neoplasia. Rev Urol. 2004 Fall;6(4):171-179.

26. Vis AN, Van Der Kwast TH. Prostatic intraepithelial neoplasia and putative precursor lesions of prostate cancer: a clinical perspective. BJU Int. 2001;88(2):147-57.

27. Nelson WG, De Marzo AM, De Weese TL. The role of inflammation in the pathogenesis of prostatic carcinoma. J Urol. 2004;172(5 Pt 2):S611; discussion S11-2.

28. Sfanos KS, De Marzo A.M. Prostate cancer and inflammation: the evidence. Histopathology. 2012;60(1):199-215. doi: 10.1111/j.13652559.2011.04033.x

29. Putzi MJ, De Marzo AM. Morphologic transition between proliferative inflammatory atrophy and high-grade intraepithelial neoplasia. Urology. 2000;56(5):828-32.

30. Sakr WA, Grignon DJ, Crissman JD, Heilbrun LK, Cassin BJ, Pontes JJ et al. In Vivo. HGPIN and prostatic adenocarcinoma between the ages of 20-69: an autopsy study of 249 cases. In Vivo. 1994;8(3):439-43.

31. Goeman L, Joniau S, Ponette D, Van der Aa F, Roskams T, Oyen R, et al. Is low-grade prostatic intraepithelial neoplasia a risk factor for cancer? Prostate Cancer and Prostatic Diseases. 2003;6:305-310. doi:10.1038/sj.pcan.4500681.

32. Breuss JM, Gallo J, DeLisser HM, Klimanskaya IV, Folkesson HG, Pittet $\mathrm{JF}$, et al. Expression of the beta6 integrin subunit in development, neoplasia and tissue repair suggests a role in epithelial remodeling. J Cell Sci. 1995;108(Pt 6):2241-51.

33. Warlick C, Feia K, Tomasini J, Iwamoto C, Lindgren B, Risk M. et al. Rate of Gleason 7 or higher prostate cancer on repeat biopsy after a diagnosis of atypical small acinar proliferation. Prostate Cancer Prostatic Dis. 2015;18(3):255-9. doi: 10.1038/pcan.2015.14.
34. Bandyopadhyay A, Raghavan S. Defining the role of integrin $\alpha \nu \beta 6$ in cancer. Curr Drug Targets. 2009;10(7):645-652.

35. Keller ET, Brown J. Prostate cancer bone metastases promote both osteolytic and osteoblastic activity. J Cell Biochem 2004;91:718-29.

36. Li J, Wang T, Goel HL, Jiang Z, Cai Y, Crockett KA, et al. A novel mechanism of prostate cancer growth mediated by $\alpha v \beta 6$ Integrin and Androgen Receptor. Presented at the First IMPaCT Meeting, Atlanta, GA, USA. 2007.

37. Naber, H P, Wiercinska E, Pardali E, van Laar, T, Nirmala E, Sundqvist A, van Dam, H, van der Horst, et al. BMP-7 inhibits TGF- $\beta$-induced invasion of breast cancer cells through inhibition of integrin $\beta 3$ expression. Cell Oncol (Dordr). 2012;35(1):19-28. doi: 10.1007/ s13402-011-0058-0.

38. Bianchi, A, Gervasi M E, Bakin A. Role of $\beta 5$-integrin in epithelialmesenchymal transition in response to TGF- $\beta$. Cell Cycle. 2010;9:1647-1659.

39. Tatler AL, John AE, Jolly, L, Habgood A, Porte J, Brightling C, et al. Integrin v $\beta 5$-mediated TGF- $\beta$ activation by airway smooth muscle cells in asthma. J Immunol. 2011;187(11):6094-107. doi: 10.4049/ jimmunol.1003507.

40. Zambruno G, Marchisio PC, Marconi A, Vaschieri C, Melchiori A, Giannetti A, et al. Transforming growth factor- $\beta 1$ modulates $\beta 1$ and $\beta 5$ integrin receptors and induces the de novo expression of the $\alpha v \beta 6$ heterodimer in normal human keratinocytes: Implications for wound healing. J. Cell Biol. 1995;129:853-865.

41. Bianchi A, Gervasi ME, Bakin A. Role of $\beta 5$-integrin in epithelialmesenchymal transition in response to TGF- $\beta$. Cell Cycle. 2010;9(8):1647-59.

42. Van der Pluijm G. Epithelial plasticity, cancer stem cells and bone metastasis formation. Bone. 2011;48(1):37-43. doi: 10.1016/j. bone.2010.07.023

43. Bandyopadhyay A, Raghavan S. Defining the Role of Integrin $\alpha v \beta 6$ in Cancer. Cur Drug Targets. Curr Drug Targets. 2009;10(7):645-652.

44. Garlick DS, Jing Li, Brian Sansoucy, Tao Wang, Leeanne Griffith, T] FitzGerald, et al. $\alpha v \beta 6$ integrin expression is induced in the POET and Ptenpc-/- mouse models of prostatic inflammation and prostatic adenocarcinoma. Am J Transl Res. 2012;4(2):165-174.

45. Bechis SK, Otsetov AG, Ge R, Olumi AF. Personalized Medicine for Management of Benign Prostatic Hyperplasia. J Urol. 2014;192(1):1623. doi: 10.1016/j.juro.2014.01.114 